\title{
Performance Evaluation of Neighbor-Awareness at the Media Access Control (MAC) Layer for Vehicular Ad-Hoc Networks (VANETs)
}

\author{
Marthinus J. Booysen and Gert-Jan van Rooyen
}

\begin{abstract}
Efficient medium access in Vehicular Ad-hoc NETworks (VANETs) remains a significant challenge. Two types of Media Access Control (MAC) approaches have been proposed, namely contention-based and contention-free. We propose a novel MAC approach called Discretized RAndom Medium Access (DRAMA) for VANET. DRAMA is based on a contentionfree MAC, but unlike existing contention-free approaches, it is neighbor-agnostic. We evaluate the effect on performance of taking away all forms of channel coordination, thereby essentially removing neighbor awareness from an existing MAC approach. The evaluation is performed in terms of packet delivery ratio, receiver throughput, and end-to-end latency. We also present a performance evaluation of DRAMA and compare it to the IEEE 802.11p standard for various traffic scenarios. The results show that removing awareness from a MAC has a similar effect on performance to reducing awareness, in terms of number of hops used. Our performance results demonstrate performance improvements of $48 \%, 130 \%$, and $73 \%$ over IEEE 802.11p in terms of packet delivery ratio, receiver throughput, and end-to-end latency, respectively.
\end{abstract}

\section{INTRODUCTION}

The field of vehicular communications has attracted substantial attention over the last few years. Vehicular Adhoc NETworks (VANETs) enable service delivery to vehicles by enabling Vehicle-to-Vehicle (V2V) and Vehicle-toInfrastructure (V2I) communications. The various applications proposed for VANET can be categorized as safetyrelated, traffic management, and service-related [1]. The requirements on the communications system for each application category are different. Safety messages require message delivery with low end-to-end latency and high delivery ratio, which in turn requires fast and uninterrupted access to the wireless medium. Traffic management transmissions, on the other hand, are less sensitive to delays and do not require a high delivery ratio [1], [2].

Access control to the shared wireless medium has proven to be a challenge for Vehicular Ad-hoc NETworks (VANETs) due to severe node mobility, varying communications environments, and variable node densities and node distributions apparent in the vehicular environment [3], [4], [2].

In this work we focus on the broadcasting of safety messages in the $\mathrm{V} 2 \mathrm{~V}$ context. For these messages, the requirement is therefore to deliver messages with a high probability of successful transmission and a low latency, in scenarios where there is no infrastructure support to coordinate communications. To support delivery of safety messages in the $\mathrm{V} 2 \mathrm{~V}$ scenario, we proposed a contention-free

M.J. Booysen and G.-J. van Rooyen are with the MIH Media Lab and the Department of Electrical and Electronic Engineering, Stellenbosch University, Stellenbosch, South Africa. mjbooysen@sun.ac.za approaches with multi-hop neighbor awareness in [5]. In this work we evaluate the effect of removing neighbor awareness, and to that end, we propose a Medium Acces Control (MAC) method, called Discretized RAndom Medium Access (DRAMA), which uses the same timing sequence as the work in [5] and [6]. A key feature of DRAMA is, however, that no attempt is made to interact or coordinate with neighboring nodes for medium access. We compare the performance of neighbor-agnostic DRAMA against a multi-hop neighboraware contention-free MAC, and against the existing MAC standard, IEEE $802.11 \mathrm{p}$, using packet delivery ratio, receiver throughput, and end-to-end latency.

The rest of this paper is organized as follows. In section II we describe recent MAC approaches for VANETs and categorize these methods. Section III presents a novel contentionfree TDMA approach called Discretized RAndom Medium Access (DRAMA). In section IV we describe the simulation setup and performance metrics used in our performance evaluation tests. Performance results are presented in Section V. Finally, section VI concludes the paper.

\section{RELATED WORK}

To support Wireless Access in the Vehicular Environment (WAVE), the IEEE has specified IEEE 802.11p [7] for Medium Access Control (MAC), and IEEE 1609.4 [8] for multi-channel access. The IEEE WAVE standards specify a contention-based protocol that prescribes how nodes compete for access to the shared wireless medium. Synchronization is achieved through an access scheme that alternates between control and safety communications in the first 50 milliseconds, and service communications in the subsequent 50 milliseconds, as illustrated by Fig. 1.

IEEE $802.11 \mathrm{p}$ has become the de facto standard MAC for use in VANETs. However, this approach could lead to unbounded medium access delays which is unacceptable for the transmission of life-critical safety messages. In the broadcast mode, IEEE $802.11 \mathrm{p}$ is also susceptible to the hidden node problem, which has a severe impact on successful message delivery. The hidden node problem describes a situation where two nodes that are in range of a third node, but out of range of each other, transmit at the same time, causing a collision at the third node.

To overcome the hidden node problem, the unicast mode in IEEE $802.11 \mathrm{p}$ requires nodes to use Request To Send/Clear To Send (RTS/CTS) handshaking before transmission is attempted. Coordination in the broadcasting mode of IEEE $802.11 \mathrm{p}$ is simpler: If a unit wants to broadcast, it waits for a period called the Arbitration Inter-Frame Space (AIFS). 
If no activity is sensed during the AIFS, the the node can transmit. However, if activity is sensed during the AIFS, the node that wants to transmit backs off for a period called the Contention Window (CW), after which the process starts again with the AIFS. The CW is based on a counter, which increases if the $\mathrm{CW}$ is entered into again before a successful transmission attempt.

To overcome the obstacles inherent to contention-based approaches, such as IEEE $802.11 \mathrm{p}$, various alternative MAC approaches have recently been proposed [9], [10], [11], [3], [12], [6]. The majority of alternative approaches are contention-free and use Time Division Multiple Access (TDMA) to coordinate access to the wireless medium. TDMA synchronization is usually achieved through Global Positioning System (GPS) time. Since infrastructure cannot be assumed to be available in the $\mathrm{V} 2 \mathrm{~V}$ scenario, MAC methods need to be self-organizing (this is an inherent feature of IEEE 802.11p). Two types of self-organization are used by contention-free MAC approaches, namely a group-based approach [9], [10], [11] and a distributed approach [3], [12], [5], [6].

In group-based approaches a leader node coordinates communication and wireless access. The leader node needs to be aware of all the nodes in the group and the group is dependent on the continued presence of the leader node, which is problematic when vehicles are highly mobile and have intermittent connectivity, as is the case in the vehicular environment. With distributed approaches, the coordination responsibility is shared among all the nodes in the network. Nodes frequently broadcast slot allocation information to notify their neighbors of their (and other nodes') slot selection. The notification is different for different approaches - a binary bit for each slot to indicate slot availability [12], or the identification numbers of the nodes owning the slots [6], [5]. The slot allocation information that each node transmits is based on information the node gathers from surrounding nodes. What constitutes "surrounding nodes" is different for different approaches and ranges from only neighbors in RF range (immediate neighbors) to multi-hop neighbors, or even accumulation with limitless number of hops [6]. Even if multi-hop neighbor awareness is used, these approaches still suffer from the hidden node problem, which is exaggerated by vehicle mobility in VANET (as nodes move in range of each other before other nodes become aware of their presence). Both the group and distributed approaches incur communications overheads and suffer from implementation complexity to maintain coordination despite node mobility.

In [13] we proposed and evaluated a contention-free TDMA-based MAC approach that uses a predetermined multi-hop awareness range to distribute MAC slot allocation information to neighboring nodes, as illustrated in Fig. 2. Nodes use the information from surrounding slots to select unused slots, thereby avoiding collisions. The multi-hop strategy is employed to overcome the hidden problem. When information from only immediate neighbors is used, i.e. zero hops, the hidden node problem severely impacts performance. For a 1-hop setup (i.e. information from immediate

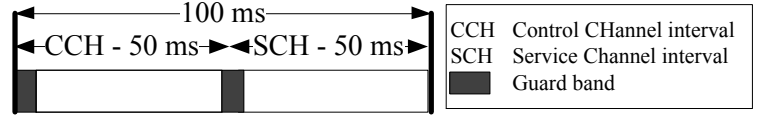

Fig. 1. Broadcasting in IEEE $802.11 \mathrm{p}$.

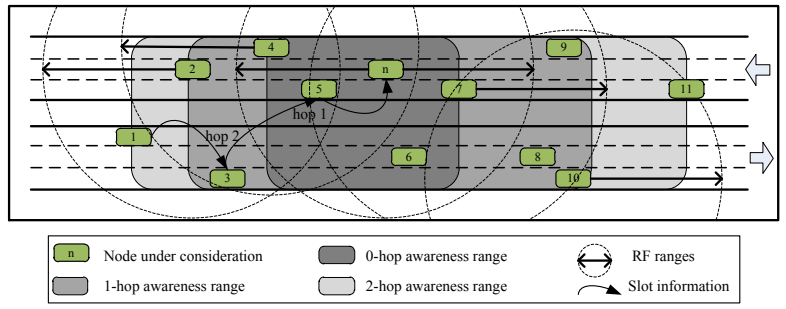

Fig. 2. Multi-hop awareness ranges.

neighbors and their neighbors) the effect of the hidden node problem on performance is reduced, but still significant. We found that the optimal performance is achieved with two hops. Further increases in hop-count results in only marginal performance improvements. Although neigbor agnostic approaches have been proposed in in [14], [15] and [16], these approaches cannot be easily modified to add awareness, since they are not variants of existing neighbor aware approaches. Since they cannot be easily extended to vary the level of awareness, it is not easy to evaluate the effect of only awareness on MAC performance. Other neighbor agnostic approaches have been proposed and discussed in [14],[15] and [16], but these are not extensions of existing neighbor aware approaches, consequently preventing and evaluation of the effect of different levels of awareness on MAC performance.

In this paper, we evaluate to what extent having no neighbor awareness impacts the performance of the MAC, by completely removing neighbor awareness from the slot selection process, while keeping the timing sequence and organizational structure of the MAC intact. The questions we ask are: How does removing awareness from an existing MAC with neighbor awareness affect performance under the extreme mobility apparent in VANET? Also, how does the resulting performance compare with the contention-based IEEE 802.11p?

\section{Proposed Contention Free Media Access CONTROL METHOD (DRAMA)}

In this section we describe our proposed MAC approach called Discretized RAndom Medium Access (DRAMA). DRAMA works on the premise that, due to severe node mobility and intermittent connectivity, it might make sense to not even attempt to coordinate slot allocations at all, and rather risk packet collisions.

To ensure that DRAMA, which has no neighbor awareness, can directly be compared to neighnor-aware MAC, DRAMA follows the algorithm in [5], except that slots are not chosen based on neighboring nodes, but at random. A TDMA frame of 50 slots, of 1 millisecond each, is 


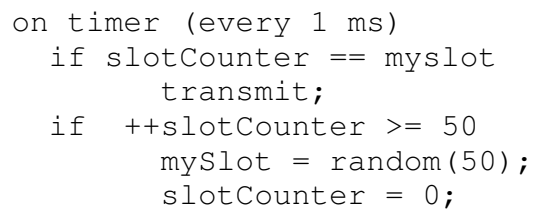

Fig. 3. Algorithm for slot allocation in DRAMA.

TABLE I

ROAD SIMULATION PARAMETERS USED.

\begin{tabular}{|l|c|c|c|}
\hline Environment & Length/Area & High density & Low density \\
\hline Highway & $1.75 \mathrm{~km}$ & $25 / \mathrm{km} /$ lane & $12 / \mathrm{km} /$ lane \\
Urban & $3 \mathrm{~km}^{2}$ & $100 / \mathrm{km}^{2}$ & $50 / \mathrm{km}^{2}$ \\
\hline
\end{tabular}

used to match WAVE's $\mathrm{CCH}$ and to enable comparison with the work in [5] and [6]. This selection also supports cooperative awareness messages of 8 kilobits at a data rate of 10 Megabits/second, in line with the WAVE standards discussed in [17]. Different to traditional contention-free methods, DRAMA has no coordination between nodes to try to coordinate communication, apart from synchronization. Collisions are therefore not avoided by selecting unused slots, meaning collisions could occur.

At the beginning of every TDMA frame, the node selects a transmission slot at random to transmit from. All slots are equally likely to be selected. This is different to Mobile Slotted ALOHA [14], to allow a direct comparison with our neighbor aware MACs, and to allow us to single out the effect of awareness on the MAC performance. Because of the simplified coordination scheme, no coordination overhead is required in the transmitted packet. In addition, no time is lost in re-allocating slots in the event of a node becoming aware of another node using the same slot, which is the case in neighbor aware MACs. The simple algorithm for DRAMA is illustrated in Fig. 3.

\section{Simulation Setup and Performance Metrics}

To accurately simulate communications in a vehicular environment, both the movement of vehicles and communications characteristics (such as signal path loss, receiver sensitivity, transmission power, etc.) need to be taken into account [18]. To simulate this, we use the OMNeT++ network simulator with the MiXiM framework [19][20]. We use real-world maps from [21] and a road traffic simulation package, called Simulation of Urban MObility (SUMO) [22]. We conducted performance evaluation tests for highway and urban driving scenarios with high density and low density road traffic. The road traffic simulation properties are listed in Table I.

Table II lists the network parameters used in our network simulation tests. Each node transmits a cooperative awareness message once per $\mathrm{CCH}$.

We use three metrics to evaluate the MAC methods for the delivery of safety messages. The first metric is the packet
TABLE II

SIMULATION PARAMETERS USED IN OUR SIMULATION TESTS.

\begin{tabular}{|l|c|c|l|}
\hline Parameter & Value & Unit & Source \\
\hline Carrier frequency & 5.9 & $\mathrm{GHz}$ & {$[7]$} \\
Transmit power & $100.0(20)$ & $\mathrm{mW}(\mathrm{dBm})$ & {$[7][17]$} \\
Bitrate & 10 & $\mathrm{Mbps}$ & {$[6][7]$} \\
Packet size & 8 & $\mathrm{kbits}$ & {$[7][8]$} \\
Path loss exponent $(\alpha)$ & 3.0 & - & {$[18][23][24]$} \\
Receiver sensitivity & -80 & $\mathrm{dBm}$ & {$[17]$} \\
Contention Window & $31-1023$ & & {$[7][17]$} \\
Simulation time & 200 & seconds & \\
\hline
\end{tabular}

delivery ratio and is defined as

$$
\rho=\frac{\sum_{i=1}^{N} r_{i}}{n_{\mathrm{RF}} \sum_{i=1}^{N} t_{i}}
$$

where $r_{i}$ is the number of packets received by node $i, t_{i}$ is the number of packets transmitted by node $i$, and $n_{\mathrm{RF}}$ is the average number of in-range RF neighbors. The value of $n_{\mathrm{RF}}$ was approximated from a geometric model of the road environment and the vehicle density. To confirm the approximation of $n_{\mathrm{RF}}$, we temporarily broke the WAVE alternating sequence and set the number of slots per TDMA frame to 1000 and allowed only every tenth node to transmit, which results in a negligible number of collisions, in which case $\rho$ should was equal to one. In essence, packet delivery ratio measures the success ratio of transmissions - the probability of successful reception for a transmitted packet, which is crucial for broadcast of safety messages.

The second metric we use is receiver throughput (the accumulated data received in the network at the application layer per second, or goodput), averaged per node, which is defined as

$$
\theta=\frac{\sum_{i=1}^{N} r_{i}}{T_{\text {sim }} N_{\text {ave }}}
$$

where $T_{\operatorname{sim}}$ is the simulation duration in seconds, and $N_{\text {ave }}$ is the average number of active vehicles in the network. In the urban scenarios the value of $N_{\text {ave }}$ is 300 and 150 for the high density and low density tests respectively, and for the highway scenarios $N_{\text {ave }}$ is 262 and 126 for the high density and low density tests respectively. Note that the total number of vehicles in the simulation is much higher $-N_{\text {ave }}$ only reflects the number of active vehicles at any given time. Receiver throughput therefore gives an indication of how much data was successfully received in the network, averaged per second and per node.

The third performance metric is the end-to-end latency, which is defined as the delay from the application-layer of the transmitting node, to the application layer of the receiving node. Latency is only calculated for packets that are received successfully. Since we are evaluating the MAC layer only, we have not incorporated retransmission attempts at the application layer, and no Time To Live (TTL) was enforced.

\section{Performance Results}

The results in Fig. 4 illustrate the receiver throughput and delivery ratio for different awareness ranges. The results 


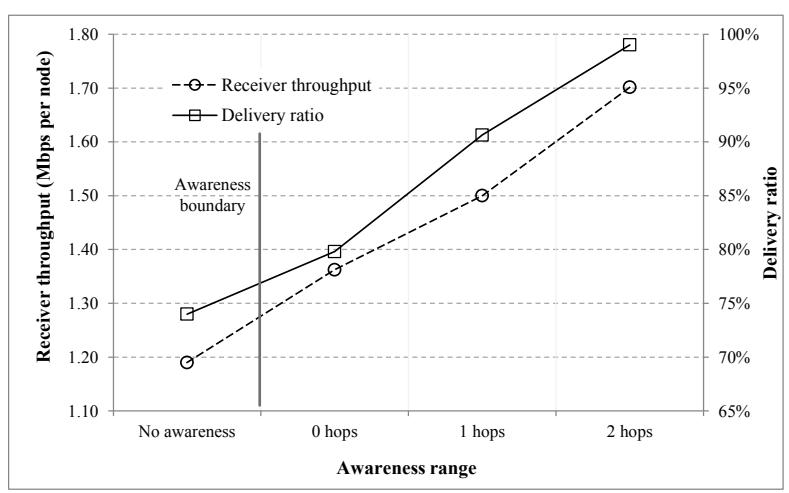

Fig. 4. Receiver throughput and delivery ratio results for different awareness ranges.

TABLE III

EFFECT OF AWARENESS RANGE ON MAC PERFORMANCE.

\begin{tabular}{|l|c|c|}
\hline Awareness & Packet delivery ratio & Receiver Throughput \\
\hline None & 1.19 & $74 \%$ \\
0 hops & 1.36 & $80 \%$ \\
1 hops & 1.50 & $91 \%$ \\
2 hops & 1.70 & $99 \%$ \\
\hline
\end{tabular}

show that a reduction in awareness leads to a reduction in both receiver throughput and delivery ratio, as expected. Receiver throughput for a neighbor agnostic MAC (DRAMA) is $9.18 \%$ less than a 0-hop MAC, where information from only the immediate in-range neighbors is used. The same can been seen in the delivery ratio - use of a 0-hop awareness range results in a delivery ratio of 6 percentage points more than the neighbor-agnostic case. The reason for the reduction in performance, for both the delivery ratio and the receiver throughput, is the increase in collisions, as more nodes transmit at the same time due to selecting the same TDMA slot to transmit in. Surprisingly, for both delivery ratio and receiver throughput, the removal of awareness has a similar effect as a single reduction in the awareness range (number of hops) from 2-hop to 1-hop, and from 1-hop to 0-hop, i.e. the effect seems to be linear through the awareness boundary (from 0-hop to no awareness). There was no significant change in end-to-end latency, since the TDMA-style structure was the same for all awareness ranges, leading to a latency of $294 \mathrm{~ms}$ - matching the TDMA frame duration and the MAC buffer length.

We also compared the performance of DRAMA against IEEE $802.11 \mathrm{p}$. The delivery ratio results in Fig. 5 show that, despite the absence of any neighbor awareness, the probability of successful packet delivery is still higher for DRAMA than for IEEE $802.11 \mathrm{p}$. In broadcasting mode, IEEE $802.11 \mathrm{p}$ is susceptible to the hidden node problem, which severely affects the delivery ratio. Although DRAMA does not take its neighbors' slots into account, the number of TDMA slots used (50) is enough to effectively minimize the probability of transmission collisions, given the number of in-range neighbors and out-of range neighbors that still cause radio interference despite being out of range.

The receiver throughput results for DRAMA and IEEE
TABLE IV

Percentage IMPROVEMENT IN DELIVERY RATIO, RECEIVER THROUGHPUT, AND END-TO-END LATENCY OF DRAMA OVER IEEE 802.11P FOR GIVEN SCENARIOS.

\begin{tabular}{|l|c|c|c|}
\hline & $\begin{array}{c}\text { Packet } \\
\text { delivery ratio }\end{array}$ & $\begin{array}{c}\text { Receiver } \\
\text { throughput }\end{array}$ & Latency \\
\hline Highway, high density & $45 \%$ & $159 \%$ & $63 \%$ \\
Highway, low density & $47 \%$ & $39 \%$ & $45 \%$ \\
Urban, high density & $40 \%$ & $130 \%$ & $73 \%$ \\
Urban, low density & $48 \%$ & $85 \%$ & $65 \%$ \\
\hline
\end{tabular}

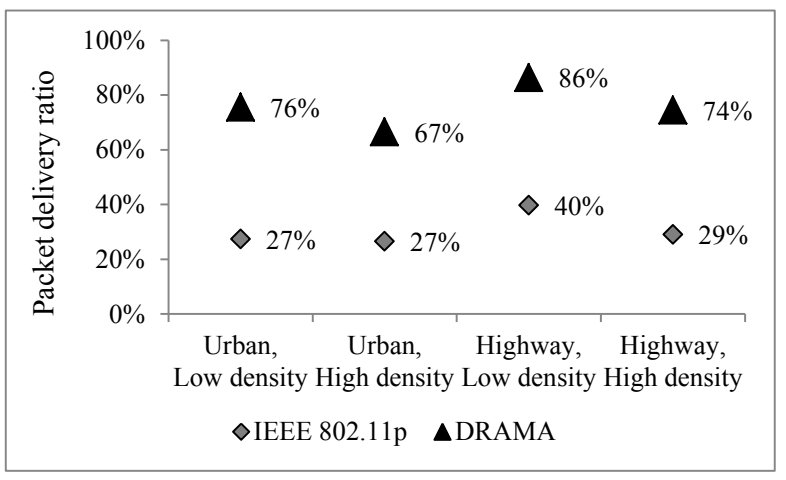

Fig. 5. Packet delivery ratio.

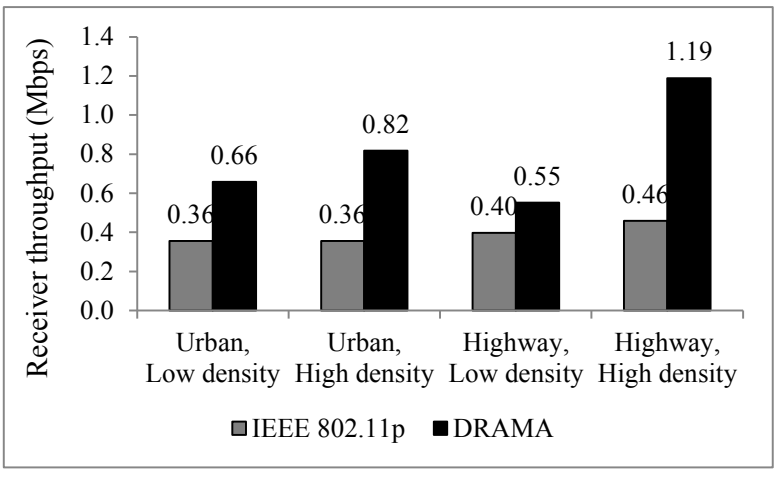

Fig. 6. Receiver throughput.

802.11p are shown in Fig. 6. From the results, we observe that more data is received per node as a result of the higher packet delivery ratio and more transmission opportunities (because the protocol does not need to contend for access to the medium) when using DRAMA.

The end-to-end latency results are shown in Fig. 7. The latencies achieved by DRAMA are lower than IEEE 802.11 , especially in the high-density scenarios, mainly because of the deterministic nature of the TDMA approach employed. Since DRAMA does not attempt to coordinate slot selection, the latency results do not depend on the node density, and is therefore highly scalable.

The improvements of DRAMA over IEEE 802.11p for the various traffic scenarios are given in Table IV.

\section{CONCLUSION}

In this work we proposed a novel contention-free TDMAbased MAC method in which slot selection is neighbor- 




Fig. 7. End-to-end latency.

agnostic. In the proposed approach, called Discretized RAndom Medium Access (DRAMA), nodes select random transmission slots before every TDMA frame. We based DRAMA on existing neighbor aware approaches to measure the impact of only removing neighbor awareness. Although the slot selection is random, nodes can only transmit in discrete slots, leading to a neighbor agnostic TDMA approach. The results show that removal of neighbor awareness completely has a similar effect to reducing the number of hops by one hop packet delivery ratio and receiver throughput is reduced. The results further show that DRAMA outperforms IEEE 802.11p in terms of receiver throughput ( $40 \%$ to $48 \%$ improvement), packet delivery ratio (39\% to $159 \%$ improvement), and endto-end latency ( $45 \%$ to $75 \%$ improvement).

\section{REFERENCES}

[1] M. Amadeo, C. Campolo, and A. Molinaro, "Enhancing IEEE 802.11p/WAVE to provide infotainment applications in VANETs," Ad Hoc Networks, vol. 10, no. 2, pp. 253-269, 2012.

[2] M. J. Booysen, S. Zeadally, and G.-J. van Rooyen, "Survey of media access control protocols for vehicular ad hoc networks," IET Communications, vol. 5, no. 11, pp. 1619-1631, February 2011.

[3] K. Bilstrup, E. Uhlemann, E. G. Ström, and U. Bilstrup, "On the ability of the 802.11p MAC method and STDMA to support real-time vehicleto-vehicle communication," EURASIP J. Wirel. Commun. Netw., vol. 2009, pp. 5:1-5:13, January 2009.

[4] P. Papadimitratos, A. La Fortelle, K. Evenssen, R. Brignolo, and S. Cosenza, "Vehicular communication systems: Enabling technologies, applications, and future outlook on intelligent transportation," IEEE Communications Magazine, vol. 47, no. 11, pp. 84-95, November 2009.

[5] M. J. Booysen, S. Zeadally, and G.-J. van Rooyen, "Performance comparison of media access control protocols for vehicular ad hoc networks," IET Networks, vol. 1, no. 1, pp. 10-19, March 2012.

[6] N. Lu, Y. Ji, F. Liu, and X. Wang, "A Dedicated Multi-Channel MAC Protocol Design for VANET with Adaptive Broadcasting," in IEEE Wireless Communications and Networking Conference: WCNC 2010 IEEE, April 2010, pp. 1-6.

[7] IEEE P802.11-REVmb/D12, November 2011 (Revision of IEEE Std 802.11-2007, as amended by IEEEs 802.11k-2008, 802.11r-2008, $802.11 y-2008,802.11 w-2009,802.11 n-2009,802.11 p-2010,802.11 z-$ 2010, 802.11v-2011, 802.11u-2011, and 802.11s-2011), title=IEEE Standard for Information technology-Telecommunications and information exchange between systems Local and metropolitan area networks-Specific requirements Part 11: Wireless LAN Medium Access Control (MAC) and Physical Layer (PHY) Specifications, pp. 1 -2910, 292012

[8] "IEEE Standard for Wireless Access in Vehicular Environments (WAVE)-Multi-channel Operation," IEEE Std 1609.4-2010 (Revision of IEEE Std 1609.4-2006), pp. 1 -89, 72011.
[9] Y. Bi, K.-H. Liu, L. Cai, X. Shen, and H. Zhao, "A multi-channel token ring protocol for QoS provisioning in inter-vehicle communications," IEEE Transactions onWireless Communications, vol. 8, no. 11, pp 5621-5631, November 2009.

[10] H. Su and X. Zhang, "Clustering-based multichannel MAC protocols for QoS provisionings over vehicular ad hoc networks," Vehicular Technology, IEEE Transactions on, vol. 56, no. 6, pp. 3309-3323, November 2007.

[11] T. Kim, S. Jung, and S. Lee, "CMMP: Clustering-Based Multi-channel MAC Protocol in VANET," in International Conference on Computer and Electrical Engineering: ICCEE 2009, vol. 1, December 2009, pp. 380-383.

[12] F. Yu and S. Biswas, "A Self Reorganizing MAC Protocol for Intervehicle Data Transfer Applications in Vehicular Ad Hoc Networks,' in International Conference on Information Technology: ICIT 2007, December 2007, pp. 110-115.

[13] M. J. Booysen, S. Zeadally, and G.-J. van Rooyen, "Impact of Neighbor Awareness at the MAC Layer in a Vehicular Ad-hoc NETwork (VANET)," IEEE International Symposium on Wireless Vehicular Communications: WIVEC2013, June 2012.

[14] H. Cozzetti and R. Scopigno, "Scalability and QoS in MS-Aloha VANETs: Forced slot re-use versus pre-emption," in IEEE International Conference on Intelligent Transportation Systems: ITSC 2011, October 2011, pp. $1759-1766$.

[15] L. Z. Michael Meisel, Vasileios Pappas, "Listen first, broadcast later: Topology-agnostic forwarding under high dynamics," in Annual Conference of International Technology Alliance in Network and Information Science., September 2010, pp. 1-8.

[16] C. Campolo, A. Molinaro, and A. Vinel, "Understanding the performance of short-lived control broadcast packets in 802.11p/WAVE Vehicular networks," in IEEE Vehicular Networking Conference: VNC 2011, nov. 2011, pp. $102-108$.

[17] J. Kenney, "Dedicated short-range communications (DSRC) standards in the United States," Proceedings of the IEEE, vol. 99, no. 7, pp. 1162-1182, July 2011.

[18] C. Sommer, D. Eckhoff, R. German, and F. Dressler, "A computationally inexpensive empirical model of IEEE $802.11 \mathrm{p}$ radio shadowing in urban environments," in International Conference on Wireless OnDemand Network Systems and Services: WONS 2011, January 2011 , pp. 84-90.

[19] "Omnet++ home page," http://www.omnetpp.org.

[20] "Mixim home page," http://mixim.sourceforge.net.

[21] "Open street maps," http://www.openstreetmap.org/

[22] "Simulation of urban mobility," http://sumo.sourceforge.net.

[23] M. Boban, O. Tonguz, and J. Barros, "Unicast communication in vehicular ad hoc networks: a reality check," Communications Letters, IEEE, vol. 13, no. 12, pp. 995 -997, december 2009.

[24] B. Blaszczyszyn, P. Muhlethaler, and Y. Toor, "Performance of MAC protocols in linear VANETs under different attenuation and fading conditions," in 12th International IEEE Conference on Intelligent Transportation Systems: ITSC 2009, oct. 2009, pp. 1 -6. 